\title{
Practical prognostic index for patients with metastatic recurrent breast cancer: retrospective analysis of 2,322 patients from the GEICAM Spanish El Alamo Register
}

\author{
Javier Puente - Sara López-Tarruella • Amparo Ruiz • Ana Lluch • \\ Miguel Pastor · Emilio Alba · Juan de la Haba - Manuel Ramos • \\ Luis Cirera • Antonio Antón - Antoni Llombart - Arrate Plazaola • \\ Antonio Fernández-Aramburo · Javier Sastre · Eduardo Díaz-Rubio • \\ Miguel Martin
}

Received: 9 December 2009/Accepted: 11 December 2009/Published online: 10 January 2010

(C) Springer Science+Business Media, LLC. 2010

\begin{abstract}
Women with recurrent metastatic breast cancer from a Spanish hospital registry (El Alamo, GEICAM) were analyzed in order to identify the most helpful prognostic factors to predict survival and to ultimately construct a practical prognostic index. The inclusion criteria covered women patients diagnosed with operable invasive breast cancer who had metastatic recurrence between 1990 and 1997 in GEICAM hospitals. Patients with stage IV breast cancer at initial diagnosis or with isolated loco-regional recurrence were excluded from this analysis. Data from 2,322 patients with recurrent breast cancer after primary treatment (surgery, radiation and systemic adjuvant
\end{abstract}

J. Puente $(\square)$ - S. López-Tarruella · J. Sastre · E. Díaz-Rubio Medical Oncology Department, Hospital Clínico San Carlos, C/Martín Lagos s/n, 28040 Madrid, Spain

e-mail: jpuente.hcsc@salud.madrid.com; docjavip@yahoo.es

\section{A. Ruiz}

Medical Oncology Department, Instituto Valenciano de

Oncologia, Valencia, Spain

\section{A. Lluch}

Medical Oncology Department, Hospital Clínico,

Valencia, Spain

M. Pastor

Medical Oncology Department, Hospital La Fe, Valencia, Spain

E. Alba

Medical Oncology Department, Hospital Virgen de la Victoria, Malaga, Spain

J. de la Haba

Medical Oncology Department, Hospital Reina Sofía, Córdoba, Spain

\section{Ramos}

Medical Oncology Department, Centro Oncológico Regional de Galicia, Corunna, Spain treatment) were used to construct the prognostic index. The prognostic index score for each individual patient was calculated by totalling up the scores of each independent variable. The maximum score obtainable was 26.1. Ninehundred and sixty-two patients who had complete data for all the variables were used in the computation of the prognostic index score. We were able to stratify them into three prognostic groups based on the prognostic index score: 322 patients in the good risk group (score $\leq 13.5$ ), 308 patients in the intermediate risk group (score 13.5115.60 ) and 332 patients in the poor risk group (score $\geq 15.61$ ). The median survivals for these groups were 3.69,

\section{Cirera}

Medical Oncology Department, Hospital Universitario Mutua de Terrassa, Terrassa, Spain

\section{A. Antón}

Medical Oncology Department, Hospital Miguel Servet,

Zaragoza, Spain

\section{A. Llombart}

Medical Oncology Department, Hospital Arnau de Vilanova,

Lleida, Spain

\section{A. Plazaola}

Medical Oncology Department, Onkologikoa, San Sebastián, Spain

A. Fernández-Aramburo

Medical Oncology Department, Complejo Hospitalario

Universitario de Albacete, Albacete, Spain

\section{Martin}

Medical Oncology Department, Hospital Gregorio Marañón, Madrid, Spain 
2.27 and 1.02 years, respectively $(P<0.0001)$. In conclusion, risk scores are extraordinarily valuable tools, highly recommendable in the clinical practice.

Keywords Prognostic factors - Metastatic breast cancer . Chemotherapy $\cdot$ Hormonal therapy

\section{Introduction}

Breast cancer is the leading cause of cancer death among women in most western countries. The annual death rate is estimated to be 40,480 women in the U.S. [1] and 5,914 in Spain [2]. MBC is incurable in most of the cases, and has a median survival of 18-24 months [3]. Therefore, treatment is usually palliative during this stage of the disease and is intended to improve the patient's quality of life and prolong survival.

Despite advances in early detection programmes and adjuvant treatments of early-stage disease, breast cancer remains as a significant health problem and patients with recurrent MBC usually die of their disease [4]. However, life expectancy of these patients varies greatly (from a few months to several years).

The prospects for $\mathrm{MBC}$ patients are generally poor, with only $10 \%$ expected to survive 10 years after diagnosis [5]. The management of these patients continues to evolve, and many new chemotherapeutic agents have become available which have improved the efficacy and tolerability of the treatments. The use of new hormonal therapies, new cytotoxic agents, the introduction of molecular targeted drugs and the different sequences of therapies administered throughout the disease have modified the natural history of MBC [6].

The value of prognostic factors in patients with earlystage breast carcinoma has been confirmed in a large number of studies. Thus, axillary nodal status, tumour size, oestrogen receptor status and histologic grade are well established [7]. In contrast, there have been only a few reports on prognostic factors in patients with $\mathrm{MBC}$ [8]. The analysis of short series indicates that the survival of patients with recurrent disease is related to the site of metastases (visceral recurrences have been associated with shorter survival), the hormonal receptor status, the adjuvant therapy received, the stage of the disease at diagnosis and the disease free interval [9].

It has been demonstrated that a small fraction of MBC patients can achieve long-term survival after systemic therapies. The identification of patients with a high or low life expectancy could have relevant therapeutic implications.

In the current study, we have analyzed women with recurrent MBC from a Spanish hospital register (El Alamo, GEICAM) in order to identify the most helpful prognostic factors to predict survival and to construct a practical prognostic index.

\section{Materials and methods}

Patients (for construction of the prognostic index)

The "Alamo Project" is a hospital registry that collects data on consecutive women diagnosed as having breast cancer in institutions belonging to GEICAM (Spanish Breast Cancer Research Group). This project is conducted in consecutive intervals of 4 years with the objective of analyzing the demography of the patients, the characteristics of their tumours, the treatment received and the clinical evolution of the disease. Data gathered in the "Alamo I \& II" projects were obtained from 50 Spanish hospitals. "Alamo I" consisted of 4,532 patients diagnosed de novo with breast cancer between 1990 and 1993. These data were collected by end of the year 2000. "Alamo II" consisted of 10,322 patients diagnosed de novo between 1994 and 1997. These data were collected by end of the year 2003. Overall, these series includes more than $10 \%$ of all breast cancer patients diagnosed in Spain during the corresponding periods of time.

There were three versions of the data collection form: data recording form on paper, electronic recording for remote access and loading into the database on SQL Server and an electronic survey form for the recording in the database using Access ${ }^{\circledR}$. Once the inclusion of the data into the database was concluded, an audit was performed to confirm the integrity, accuracy and coherence of the data. This involved selecting 100 forms at random (ensuring at least three from each participating hospital) and the data was manually checked with that in the database. The percentage discordance was $<5 \%$ of the total data compared [10].

Protection of personal data was applied in accordance with the requirements of Spanish legislation.

The inclusion criteria covered women patients diagnosed of operable invasive breast cancer between 1990 and 1997 in GEICAM hospitals who have had metastatic recurrence. Patients with stage IV at diagnosis or with isolated locorregional recurrence were not included in this analysis. Patients with a previous neoplasm except for in situ breast cancer, basal cell or squamous cell carcinoma of the skin or in situ carcinoma of the cervix appropriately treated were excluded from the analysis. Those who developed secondary neoplasms or second primary breast cancer during the follow-up period were also excluded.

Statistical analysis

Data from patients with recurrent breast cancer after primary treatment (surgery, radiation and systemic adjuvant 
treatment) were used to construct the prognostic index. The main endpoint was survival from the time of metastatic recurrence.

This study was designed as descriptive, retrospective and transversal. Qualitative variables were expressed as the absolute and relative frequencies and quantitative data were expressed as the means of central tendency and of dispersion (mean, median, trends and range). Survival rates were calculated using the Kaplan and Meier method. All deaths were considered as events, regardless of their cause. Each patient was considered to be alive at the time of last evaluation unless death had been documented.

Both a univariate and a multivariate approaches were used for the analysis of potential prognostic factors. The Cox proportional hazards model was applied for the selection of the most significant variables contributing to survival. Differences with $P$-value less than 0.05 were considered significant. All $P$-values were two-sided. Potential prognostic factors were analyzed in a multivariate analysis using a forward step-wise conditional Cox proportional hazards model.

For the construction of the prognostic index, the values of the $\beta$ coefficients of the Cox model were calculated. The results obtained from all of the significant factors in the Cox model were added together for each patient.

All analyses were performed using SPSS version 12.0 statistical software (SPSS Inc, Chicago, IL). The prognostic index was applied to the patients and overall survival rates were calculated in order to create three different prognostic groups.

We have followed the reporting recommendations for tumour marker prognostic studies (REMARK) published in 2006 [11]. These guidelines recommend elements and formats for these kind of studies, and the goal of these guidelines is to encourage transparent and complete reporting so that the relevant information will be available to others to help them to judge the usefulness of the data.

\section{Results}

Data from 2322 patients with recurrent breast cancer after primary treatment (surgery, radiation and systemic adjuvant treatment) who had been accrued between January 1990 and December 1997 on the "Alamo" projects were used to construct the prognostic index. The median followup time of the whole population was 7 years (range, 2132 months). The median overall survival was 1.8 years form the time of recurrence. Table 1 shows the variables that showed a statistically significant influence on survival in the univariate analysis.

These variables were selected for a multivariate regression analysis using the Cox proportional hazards model. Nine-hundred and sixty-two patients with complete information in all significant variables were included in the multivariate analysis. The distributions of main variables were similar in the cohort of patients included in the multivariate analysis and in the remaining patients. Stepwise regression procedures were applied to calculate the values of the $\beta$ coefficients of the Cox model, which are listed in Table. 2 .

Age and pathological state/stage of disease at diagnosis, histological grade, hormonal receptor status, nodal ratio, administration of neo and/or adjuvant chemotherapy, dominant site of metastasis, number of hormonal lines in metastatic disease and response to the first line therapy remained significantly related to survival at the end of the multivariate analysis (Table 3).

A quantitative score derived from the regression coefficients of each independent prognostic variable was created as described earlier. The prognostic index score for each individual patient was calculated by totalling up the scores of each independent variable. The maximum score obtainable was 26.1. Nine-hundred and sixty-two patients who had complete data of all variables were used for the computation of prognostic index score. We were able to stratify them into three prognostic groups based on the prognostic index score: 322 patients in the good risk group (score $\leq 13.5$ ), 308 patients in the intermediate risk group (score 13.51-15.60) and 332 patients in the poor risk group (score $\geq 15.61$ ). The median survivals for these groups were 3.69, 2.27 and 1.02 years, respectively $(P<0.0001)$. The area under ROC curve (AUC) for the nomogram was 0.694 (0.661-0.728). The survival curves stratified by prognostic score index groups are depicted in Fig. 1.

\section{Discussion}

Despite the advances accomplished in the adjuvant treatment of breast cancer, nearly a $20 \%$ node-negative and $60 \%$ node-positive breast cancer patients will recur from their disease. Recurrence location has clearly shown to be a key prognostic factor for MBC patients in terms of overall survival. Thus, visceral involvement correlates with a shorter survival than soft tissue limited disease. Other factors reported in the literature with prognostic impact have been tumour size and stage at diagnosis, disease-free survival, hormone receptor status or the adjuvant regimen received.

A Spanish report on $439 \mathrm{MBC}$ patients with a median follow-up of 30 months from the diagnosis of recurrence [9], showed that three variables were independently associated with better survival: bone or soft-tissue only metastasis, achieving an objective response (partial response + complete response) to the first-line treatment 
Table 1 Univariate survival analysis

\begin{tabular}{|c|c|c|c|c|}
\hline Variable & $N$ & Median survival (CI 95\%) & 2-year OS (CI 95\%) & $P$ (Breslow) \\
\hline Age at diagnosis & & & & $<0.001$ \\
\hline$<35$ years & 168 & $2.68(2.17-3.19)$ & $59.50 \%(63.44-55.56)$ & \\
\hline $36-50$ years & 671 & $2.07(1.87-2.27)$ & $51.83 \%(53.90-49.76)$ & \\
\hline $51-75$ years & 1297 & $1.87(1.71-2.04)$ & $46.89 \%(48.40-45.38)$ & \\
\hline$>75$ years & 152 & $0.90(0.71-1.09)$ & $21.82 \%(25.97-17.67)$ & \\
\hline Menopausal status at diagnosis & & & & 0.0181 \\
\hline Premenopausal & 797 & $2.13(1.90-2.35)$ & $52.75 \%(54.64-50.86)$ & \\
\hline Postmenpausal & 1466 & $1.76(1.62-1.90)$ & $45.13 \%(47.35-44.51)$ & \\
\hline Pathological stage at diagnosis & & & & $<0.001$ \\
\hline I & 189 & $3.08(2.64-3.51)$ & $65.41 \%(69.20-61.62)$ & \\
\hline II & 1298 & $2.11(1.94-2.28)$ & $51.25 \%(52.75-49.75)$ & \\
\hline III & 703 & $1.39(1.21-1.57)$ & $37.53 \%(39.52-35.54)$ & \\
\hline $\mathrm{T}$ of the TNM classification & & & & $<0.001$ \\
\hline $\mathrm{T} 0-\mathrm{T} 1$ & 456 & $2.55(2.25-2.85)$ & $58.84 \%(61.35-56.33)$ & \\
\hline $\mathrm{T} 2$ & 1095 & $1.99(1.81-2.17)$ & $49.80 \%(51.43-48.17)$ & \\
\hline $\mathrm{T} 3$ & 301 & $1.63(1.30-1.96)$ & $42.33 \%(45.41-39.25)$ & \\
\hline $\mathrm{T} 4$ & 394 & $1.28(1.04-1.51)$ & $35.11 \%(37.74-32.48)$ & \\
\hline $\mathrm{N}$ of the TNM classification & & & & $<0.001$ \\
\hline No & 614 & $2.43(2.07-2.79)$ & $55.82 \%(58.00-53.64)$ & \\
\hline N1 & 1357 & $1.89(1.73-2.04)$ & $47.58 \%(49.05-46.11)$ & \\
\hline $\mathrm{N} 2$ & 199 & $1.13(0.87-1.39)$ & $29.95 \%(33.48-26.42)$ & \\
\hline N3 & 3 & - & $66.67 \%(100.0-13.32)$ & \\
\hline Histologic subtype & & & & 0.0273 \\
\hline Ductal & 1880 & $1.86(1.72-2.00)$ & $47.79 \%(49.03-46.55)$ & \\
\hline Lobulillar & 186 & $2.42(2.08-2.76)$ & $59.66 \%(63.57-55.75)$ & \\
\hline Others & 177 & $1.57(1.18-1.96)$ & $39.73 \%(43.77-35.69)$ & \\
\hline Histologic grade & & & & $<0.001$ \\
\hline Grade 1 & 169 & $2.86(2.17-3.55)$ & $60.48 \%(64.65-56.31)$ & \\
\hline Grade 2 & 807 & $2.27(2.05-2.48)$ & $53.99 \%(55.89-52.09)$ & \\
\hline Grade 3 & 517 & $1.48(1.28-1.69)$ & $39.82 \%(42.15-37.49)$ & \\
\hline Hormonal receptor status & & & & $<0.001$ \\
\hline Positive & 1088 & $2.45(2.25-2.65)$ & $57.44 \%(59.06-55.82)$ & \\
\hline Negative & 445 & $1.33(1.11-1.55)$ & $36.12 \%(38.60-33.64)$ & \\
\hline Vessel permeation & & & & $<0.001$ \\
\hline Yes & 559 & $1.55(1.37-1.73)$ & $42.74 \%(44.99-40.49)$ & \\
\hline No & 1049 & $1.94(1.75-2.13)$ & $48.93 \%(50.62-47.24)$ & \\
\hline Extracapsular extension & & & & $<0.001$ \\
\hline Yes & 525 & $1.33(1.16-1.51)$ & $37.48 \%(39.76-35.20)$ & \\
\hline No & 1069 & $2.21(2.00-2.42)$ & $52.84 \%(54.50-51.18)$ & \\
\hline Surgery at diagnosis & & & & 0.0369 \\
\hline No & 22 & $0.83(0.00-1.79)$ & $23.68 \%(45.71-01.65)$ & \\
\hline Yes & 2269 & $1.90(1.78-2.02)$ & $48.16 \%(49.29-47.03)$ & \\
\hline Nodal ratio & & & & $<0.001$ \\
\hline$<0.25$ & 1095 & $2.31(2.09-2.53)$ & $54.40 \%(56.04-52.76)$ & \\
\hline$>0.25-<0.75$ & 641 & $1.78(1.57-1.99)$ & $45.81 \%(47.94-43.68)$ & \\
\hline$>0.75$ & 385 & $1.25(1.02-1.48)$ & $35.57 \%(38.17-32.97)$ & \\
\hline Neo and/or adjuvant chemotherapy with anthracyclines & & & & 0.0033 \\
\hline No & 1248 & $2.05(1.87-2.22)$ & $50.58 \%(52.13-49.03)$ & \\
\hline
\end{tabular}


Table 1 continued

\begin{tabular}{|c|c|c|c|c|}
\hline Variable & $N$ & Median survival (CI 95\%) & 2-year OS (CI 95\%) & $P$ (Breslow) \\
\hline Yes & 1043 & $1.75(1.60-1.90)$ & $45.00 \%(46.64-43.36)$ & \\
\hline Neo and/or adjuvant chemotherapy with taxanes & & & & 0.0133 \\
\hline No & 2274 & $1.89(1.77-2.02)$ & $48.08 \%(49.21-46.95)$ & \\
\hline Yes & 17 & $0.85(0.74-0.95)$ & $29.73 \%(42.08-17.38)$ & \\
\hline Adjuvant treatment with high-dose chemotherapy & & & & 0.0017 \\
\hline No & 2138 & $1.93(1.80-2.05)$ & $48.51 \%(49.68-47.34)$ & \\
\hline Yes & 153 & $1.49(1.14-1.85)$ & $40.41 \%(44.71-36.11)$ & \\
\hline Neo and/or adjuvant chemotherapy with CMF & & & & $<0.001$ \\
\hline No & 1615 & $1.76(1.63-1.88)$ & $45.49 \%(46.83-44.15)$ & \\
\hline Yes & 676 & $2.29(2.02-2.56)$ & $53.95 \%(56.04-51.86)$ & \\
\hline Disease-free interval & & & & $<0.001$ \\
\hline$<24$ months & 914 & $1.23(1.08-1.38)$ & $35.82 \%(37.48-34.16)$ & \\
\hline$\geq 24$ months & 1337 & $2.38(2.18-2.57)$ & $56.62 \%(58.10-55.14)$ & \\
\hline Menopausal status at recurrence & & & & 0.001 \\
\hline Premenopausical & 418 & $2.42(2.11-2.73)$ & $55.71 \%(58.25-53.17)$ & \\
\hline Postmenopausical & 1742 & $1.83(1.69-1.98)$ & $46.37 \%(47.68-45.06)$ & \\
\hline Number of recurrence sites & & & & $<0.001$ \\
\hline Isolated & 1340 & $2.27(2.07-2.46)$ & $54.12 \%(55.58-52.66)$ & \\
\hline Multiple & 951 & $1.49(1.31-1.68)$ & $39.01 \%(40.75-37.27)$ & \\
\hline Dominant site of metastasis & & & & $<0.001$ \\
\hline Soft tissues & 92 & - & $75.98 \%(80.82-71.14)$ & \\
\hline Bone & 827 & $2.55(2.30-2.80)$ & $59.54 \%(61.38-57.70)$ & \\
\hline Lung nodules & 347 & $2.21(1.94-2.48)$ & $54.09 \%(56.97-51.21)$ & \\
\hline Visceral & 1025 & $1.15(1.02-1.27)$ & $33.72 \%(35.34-32.10)$ & \\
\hline Surgery at recurrence & & & & $<0.001$ \\
\hline No & 2126 & $1.79(1.67-1.90)$ & $45.88 \%(47.05-44.71)$ & \\
\hline Yes & 165 & $3.88(3.03-4.73)$ & $75.05 \%(78.68-71.42)$ & \\
\hline Radiotherapy at recurrence & & & & 0.0038 \\
\hline No & 1779 & $1.82(1.68-1.96)$ & $46.51 \%(47.79-45.23)$ & \\
\hline Yes & 512 & $2.13(1.92-2.33)$ & $52.92 \%(55.28-50.56)$ & \\
\hline Antioestrogen treatment in metastatic disease & & & & $<0.001$ \\
\hline No & 1732 & $1.66(1.53-1.79)$ & $46.43 \%(44.76-42.16)$ & \\
\hline Yes & 559 & $2.79(2.50-3.07)$ & $61.37 \%(63.56-59.18)$ & \\
\hline Aromatase inhibitors in metastatic disease & & & & $<0.001$ \\
\hline No & 1479 & $1.22(1.11-1.33)$ & $34.93 \%(36.29-33.57)$ & \\
\hline Yes & 812 & $3.24(2.96-3.52)$ & $70.74 \%(72.46-69.02)$ & \\
\hline Number of hormonal therapy lines in metastatic disease & & & & $<0.001$ \\
\hline None & 937 & $1.01(0.91-1.12)$ & $29.85 \%(31.49-28.21)$ & \\
\hline 1 & 1018 & $2.23(2.06-2.40)$ & $54.48 \%(56.20-52.76)$ & \\
\hline 2 or more & 336 & $3.38(3.07-3.69)$ & $74.95 \%(79.71-70.19)$ & \\
\hline Anthracyclines treatment in metastatic disease & & & & $<0.001$ \\
\hline No & 1531 & $1.57(1.43-1.70)$ & $42.85 \%(44.24-41.46)$ & \\
\hline Yes & 760 & $2.35(2.15-2.54)$ & $57.73 \%(59.63-55.83)$ & \\
\hline Taxanes treatment in metastatic disease & & & & $<0.001$ \\
\hline No & 1485 & $1.48(1.33-1.63)$ & $41.19 \%(42.60-39.78)$ & \\
\hline Yes & 806 & $2.49(2.31-2.66)$ & $59.65 \%(61.48-57.82)$ & \\
\hline Vinca alkaloids treatment in metastatic disease & & & & 0.0457 \\
\hline No & 1686 & $1.74(1.59-1.90)$ & $45.91 \%(47.25-44.57)$ & \\
\hline
\end{tabular}


Table 1 continued

\begin{tabular}{|c|c|c|c|c|}
\hline Variable & $N$ & Median survival (CI 95\%) & 2-year OS (CI 95\%) & $P$ (Breslow) \\
\hline Yes & 605 & $2.17(1.96-2.37)$ & $53.83 \%(57.91-49.75)$ & \\
\hline Capecitabine treatment in metastatic disease & & & & $<0.001$ \\
\hline No & 2231 & $1.84(1.73-1.96)$ & $47.15 \%(49.36-44.93)$ & \\
\hline Yes & 60 & $4.38(3.21-5.55)$ & $87.12 \%(96.04-78.20)$ & \\
\hline Gemcitabine treatment in metastatic disease & & & & $<0.001$ \\
\hline No & 2195 & $1.84(1.71-1.96)$ & $46.50 \%(47.66-45.34)$ & \\
\hline Yes & 96 & $3.12(2.64-3.59)$ & $78.12 \%(82.45-73.79)$ & \\
\hline High-dose chemotherapy in metastatic disease & & & & $<0.001$ \\
\hline No & 2208 & $1.83(1.71-1.95)$ & $46.82 \%(47.97-45.67)$ & \\
\hline Yes & 83 & $4.03(3.18-4.88)$ & $85.97 \%(89.90-82.04)$ & \\
\hline Number of chemotherapy agents in metastatic disease & & & & $<0.001$ \\
\hline None & 998 & $1.34(1.15-1.54)$ & $39.92 \%(41.67-38.17)$ & \\
\hline 1 & 533 & $1.66(1.47-1.86)$ & $43.64 \%(48.11-39.17)$ & \\
\hline 2 & 500 & $2.20(1.94-2.47)$ & $54.05 \%(56.43-51.67)$ & \\
\hline 3 or more & 260 & $3.10(2.79-3.42)$ & $73.62 \%(79.09-68.15)$ & \\
\hline Response to the first line of treatment & & & & $<0.001$ \\
\hline Complete response & 178 & $4.95(3.40-6.50)$ & $82.47 \%(88.49-76.45)$ & \\
\hline Partial response & 462 & $2.58(2.37-2.80)$ & $61.79 \%(66.49-57.09)$ & \\
\hline Stable disease & 442 & $2.49(2.22-2.76)$ & $59.65 \%(64.61-54.69)$ & \\
\hline Progressive disease & 663 & $1.15(1.00-1.30)$ & $32.33 \%(34.25-30.41)$ & \\
\hline No evaluable or unknown & 316 & $1.73(1.04-2.42)$ & $47.30 \%(50.47-44.13)$ & \\
\hline
\end{tabular}

strategy and DFS over 2 years. Five-year OS was 35\% in responders and virtually zero in non-responders. The value of these three prognostic variables has been confirmed in our series. In our series, patients with skin and soft-tissue recurrences had not reached the median survival time yet, while those patients with complete response to the first-line treatment for metastatic disease had a median survival time of 4.95 years.

An American study coincided to show DFS and the recurrence location, together with the hormone receptor status, as significant prognostic factors. The median OS was 15 months in patients with HR-negative tumours, visceral metastasis and DFS less than 2 years versus 90 months in patients with HR-positive tumours, soft-tissue metastasis and DFS over 2 years [12]. In our experience, the HR-status demonstrated independent prognostic value, hence $57.44 \%$ of the HR-positive patients remained alive at 2 years versus $36.12 \%$ in the HR-negative group.

The value of lymph node involvement at diagnosis as a prognostic factor of survival in patients with recurrent disease is a matter of controversy. Chang et al. [13] analyzed the primary tumour characteristics of node-positive patients who have recurred of the disease. According to their data, tumour size and number of lymph nodes affected were crucial to the prediction of the risk of recurrence, but did not correlate with survival once metastasis had already appeared. In the multivariate model, older age ( $>50$ years),
DFS less than a year, PR-negative status, high S-phase fraction (SPF) and visceral disease were significant predictors of lower survival. On the contrary, in the experience reported by Insa et al. [9], either tumour size or number of lymph nodes involved at diagnosis had independent prognostic significance. In the Alamo database, lymph node involvement, considered as nodal ratio (absolute number of involved nodes-number of nodes resected [14]), turned out to be a relevant prognostic factor in breast cancer patients with distant metastasis. As a result, for patients with $<0.25$ ratio median survival was 2.31 years, versus 1.78 years for those whose ratio was between 0.25 and 0.75 , and 1.25 for ratios $>0.75$.

As we noted in the introduction, all the gathered evidences from literature do not clarify the potential prognostic value of age. In this Spanish series, the differences among the $59.5 \%$ of young patients ( $<35$ years-old) alive at 2 years, the $51.83 \%$ between 36 and 50 , the $46.89 \%$ between 51 and 75 and the $21.82 \%$ over 75 years-old were statistically significant $(P<0.001)$. This observation is coincident with Chang's previous finding of worsening survival at older ages.

Regarding the prognostic impact of the metastatic location, some authors classify the metastatic involvement in two prognostic subgroups of low and high risk in order to highlight this particular aspect. The most favourable profile includes skin, subcutaneous, bone and lymph node 
Table 2 Multivariate survival analysis using Cox's model

\begin{tabular}{|c|c|c|c|c|c|c|}
\hline Variable & Coefficient $(\beta)$ & SE & Wald & HR & $95 \% \mathrm{CI}$ & $P$-value \\
\hline Age at diagnosis & & & 13.794 & & & $<0.003$ \\
\hline \multicolumn{7}{|l|}{$<35$ years } \\
\hline $36-50$ years & 0.344 & 0.224 & 2.358 & 1.411 & $0.91-2.19$ & 0.125 \\
\hline $51-75$ years & 0.327 & 0.221 & 2.182 & 1.387 & $0.90-2.14$ & 0.14 \\
\hline$>75$ years & 1.087 & 0.313 & 12.089 & 2.964 & $1.61-5.47$ & 0.001 \\
\hline Stage at diagnosis & & & 5.565 & & & 0.062 \\
\hline \multicolumn{7}{|l|}{ I } \\
\hline II & 0.385 & 0.192 & 4.005 & 1.47 & $1.01-2.14$ & 0.045 \\
\hline III & 0.504 & 0.213 & 5.565 & 1.655 & $1.10-2.51$ & 0.018 \\
\hline Histological grade & & & 10.109 & & & 0.006 \\
\hline \multicolumn{7}{|l|}{ I } \\
\hline II & 0.243 & 0.169 & 2.071 & 1.275 & $0.92-1.78$ & 0.015 \\
\hline III & 0.492 & 0.175 & 7.892 & 1.635 & $1.12-2.30$ & 0.005 \\
\hline Hormonal receptor status & 0.265 & 0.11 & 5.769 & 1.304 & $1.05-1.62$ & 0.016 \\
\hline Extracapsular extension & 0.139 & 0.116 & 1.421 & 1.149 & $0.91-1.44$ & 0.233 \\
\hline Nodal ratio & & & 6.702 & & & 0.035 \\
\hline \multicolumn{7}{|l|}{$\leq 0.25$} \\
\hline $0.25-0.75$ & 0.285 & 0.125 & 5.191 & 1.329 & $1.04-1.70$ & 0.023 \\
\hline$>0.75$ & 0.346 & 0.16 & 4.642 & 1.413 & $1.03-1.93$ & 0.031 \\
\hline Neo and/or adjuvant chemotherapy & -0.32 & 0.131 & 5.999 & 0.726 & $0.56-0.94$ & 0.014 \\
\hline Surgery at recurrence & -0.178 & 0.214 & 0.693 & 0.837 & $0.55-1.27$ & 0.405 \\
\hline Radiotherapy at recurrence & 0.117 & 0.119 & 0.966 & 1.124 & $0.90-1.42$ & 0.326 \\
\hline Dominant site of metastasis & & & 27.499 & & & $<0.001$ \\
\hline \multicolumn{7}{|l|}{ Soft tissues } \\
\hline Bone & 1.038 & 0.386 & 7.224 & 2.823 & $1.32-6.01$ & 0.007 \\
\hline Lung nodules & 1.373 & 0.391 & 12.358 & 3.949 & $1.84-8.50$ & $<0.001$ \\
\hline Visceral & 1.493 & 0.377 & 15.659 & 4.451 & $2.12-9.32$ & $<0.001$ \\
\hline Number of hormonal therapy lines in metastatic disease & & & 29.355 & & & $<0.001$ \\
\hline None & 0.875 & 0.169 & 26.686 & 2.398 & $1.72-3.34$ & $<0.001$ \\
\hline 1 & 0.418 & 0.152 & 7.574 & 1.519 & $1.13-2.05$ & 0.006 \\
\hline \multicolumn{7}{|l|}{2 or more } \\
\hline Number of chemotherapy agents in metastatic disease & & & 3.872 & & & 0.276 \\
\hline None & 0.262 & 0.165 & 2.525 & 1.3 & $0.94-1.80$ & 0.112 \\
\hline 1 & 0.301 & 0.158 & 3.634 & 1.351 & $1.00-1.84$ & 0.057 \\
\hline 2 & 0.189 & 0.155 & 1.498 & 1.209 & $0.89-1.64$ & 0.221 \\
\hline \multicolumn{7}{|l|}{3 or more } \\
\hline Response to the first line therapy & & & 61.185 & & & $<0.001$ \\
\hline \multicolumn{7}{|l|}{ Complete response } \\
\hline Partial response & 0.548 & 0.242 & 5.13 & 1.73 & $1.01-2.80$ & 0.03 \\
\hline Stable disease & 0.685 & 0.24 & 8.129 & 1.984 & $1.24-3.12$ & 0.001 \\
\hline Progressive disease & 1.317 & 0.234 & 31.632 & 3.733 & $2.36-5.91$ & $<0.001$ \\
\hline No evaluable or unknown & 0.766 & 0.263 & 8.496 & 2.151 & $1.28-3.60$ & $<0.001$ \\
\hline
\end{tabular}

metastasis together with nodular lung metastasis, and is characterized by a slower growth rate and a longer survival. In contrast, brain, liver and multiple lung metastasis, carcinomatous lymphangitis or massive pleural effusions are closely linked to a worse outcome. Almost all the multivariate analyses agree to emphasize that liver disease is a predictor of poor response to chemotherapy and a marker of adverse outcome, even if this is the only location of the disease. Our study is fully consistent with this reality so that median survival of $\mathrm{MBC}$ patients with visceral 
Table 3 Prognostic index score

\begin{tabular}{ll}
\hline Variable & $\beta$-value \\
\hline
\end{tabular}

Age at diagnosis

$<35$ years

0

$36-50$ years

1.4

$51-75$ years

1.4

$>75$ years

3

Stage at diagnosis

I

II

III

1.7

Hormonal receptor status

$\mathrm{RE}$ and/or RP positive

$\mathrm{RE}$ and RP negative

Histological grade

I

0

II

1.3

III

Extracapsular extension

No

Yes

Nodal ratio

$\leq 0.25$

$0.25-0.75$

$>0.75$

Neo and/or adjuvant chemotherapy

No

Yes

Dominant site of metastasis

Soft tissues 0

Bone $\quad 2.8$

Lung nodules $\quad 3.9$

Visceral 4.5

Number of recurrence sites

Isolated

$$
0
$$

Multiple

Surgery at recurrence

No $\quad 0$

Yes $\quad 0.8$

Radiotherapy at recurrence

$\begin{array}{ll}\text { No } & 0 \\ \text { Yes } & 1.1\end{array}$

Number of chemotherapy agents in metastatic disease

0

1

2

3 or more

Number of hormonal therapy lines in metastatic disease

$\begin{array}{ll}0 & 2.4 \\ 1 & 1.5\end{array}$

Table 3 continued

\begin{tabular}{ll}
\hline Variable & $\beta$-value \\
\hline 2 or more & 0 \\
Response to the first line therapy & \\
Complete response & 0 \\
Partial response & 1.7 \\
Stable disease & 1.9 \\
Progresive disease & 3.7 \\
No evaluable or unknown & 2.2 \\
\hline
\end{tabular}

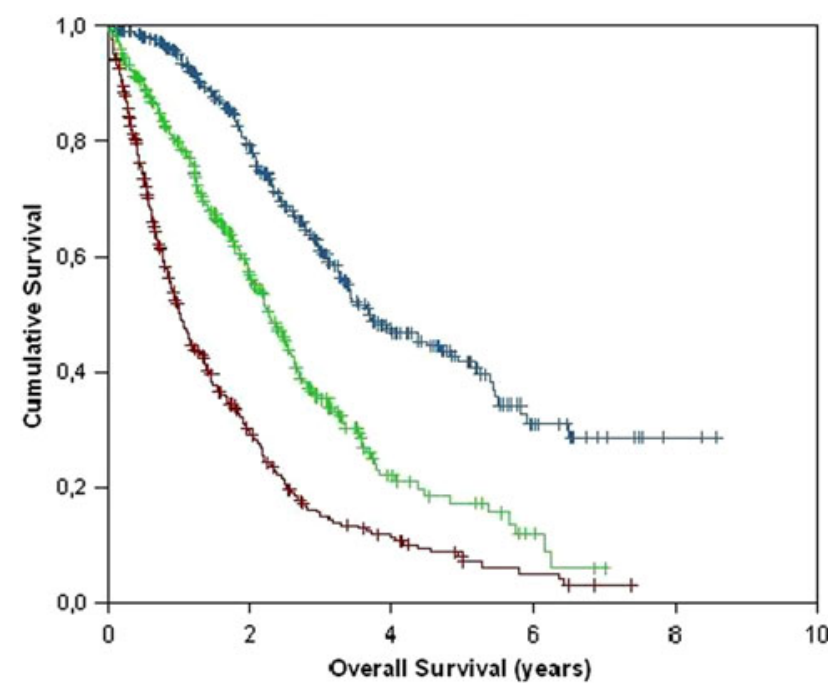

Fig. 1 Comparative survival curves according to the prognostic index. The blue curve represents the good risk group (score $\leq 13.5$ ), the green curve represents the intermediate risk group (score 13.5115.60) and the red curve represents the poor risk group (score $\geq 15.61)$. The median survivals for these groups were 3.69, 2.27 and 1.02 years, respectively, $(P<0.0001)$

involvement (mainly liver metastases) is 1.15 years, versus the 2.21 years if patients have nodular lung metastasis, 2.55 years in the case of bone metastasis and not even reached median survival if skin and/or soft-tissue metastasis is detected.

The number of organs involved is also correlated with OS. The Alamo project analysis showed $54.12 \%$ of the patients were alive at 2 years if only one organ was affected by metastasis, which decreased to $39.01 \%$ in the case of multiple organ involvement. In a previous study of patients with MBC including the bone, median OS was nearly 2.6 years when this was the only location of the disease and dropped to 1 year if a second metastatic location existed [15]. The data from the previously mentioned study of Insa et al. [9], demonstrated a 30 months OS for the single location group, 22 months for two metastatic locations and 11 months if there were three or more locations. Conversely, the EORTC joint analysis showed 
22-26 months of OS for patients with exclusive liver involvement and 14-16 months if the disease was scattered in several locations [16].

In view of our results, the treatment regimen administered to MBC patients has a potential prognostic impact. First of all, a worse survival was found for those patients who received adjuvant or neoadjuvant treatment, specially if it was an anthracycline-based regimen. This fact is consistent with the published evidence, such as the results of the Spanish study carried out over 297 MBC patients, which showed a more adverse outcome for patients with multiple metastatic involvement, a short DFS interval and previous anthracycline-based chemotherapy regimes [17]. A plausible explanation is that these treatments may select resistant clones or may introduce de novo mutations leading to a more aggressive metastatic disease behaviour.

Regarding the role of local therapies such as surgery or radiotherapy, there are no available randomized clinical trial data demonstrating the benefit of a surgical resection of metastasis over systemic therapies, so that there is no conclusive evidence at this point. However, some observational studies have provided some valuable information showing long-survival outcomes after the resection of solitary metastasis, mainly in good performance status and long disease-free interval patients [18]. It is likely that our results reflect this tendency and that probably those patients surgically managed in our series shared a more favourable prognostic profile.

Finally, two histopathologic aspects showed prognostic relevance in our experience. Histopathologic grade, extracapsular extension and tumoural stage at first diagnosis are established prognostic factors in the therapeutic decision algorithm of early breast cancer. In contrast, their prognostic significance in the metastatic setting is not so well defined in the literature. The GEICAM series showed 3.08 years median survival for stage I patients after recurrence versus 1.39 years for stage III $(P<0.001)$, 2.86 years median survival for grade I tumours versus 1.48 for grade III ones $(P<0.001)$, and 2.21 years median survival for patients with extracapsular extension versus 1.33 if they do not present this characteristic.

The development of a prognostic model starts with the precise definition of the result variable, usually death, and the analysis of the potential prognostic factors related to it. It has always been a question of remarkable interest for human beings to predict future events in advance, in fact, prophets enjoyed a bunch of privileges when the world was controlled by uncertainty and foretelling. As humanity evolved, humans looked for the way to improve their prediction methods overcoming their insecurity. Medicine did not skip this phenomenon and has been always focused in the prediction of patients outcome.
Once a model is built, it requires a validation phase that involves different steps to confirm that the model is reliable and robust. From this perspective, the qualification of the discrimination ability of the models depends on the area under the ROC curve. Our study reached the 0.69 value for distant disease. Note that even when these models have a high discrimination ability, the value will never be over 1 . For this reason, the use of a predictive score in a particular patient has a purely orientative value. A particular risk can be estimated but we could never predict the final result in this case. In other words, models with very good predictive ability can estimate a mortality, for example, of five out of 100 patients with a small error, but will not be able to determine which five patients will die.

Another big weakness that has been suggested is that scores are the representation of a snapshot from the clinical practice in an specific moment. In the Alamo series, the practice took place between 1990 and 1997 and is quite different in some important aspects form the current clinical scope. New drugs have recently joined the therapeutic store such as anti-angiogenic drugs (bevacizumab), monoclonal antibodies targeting HER2 (trastuzumab) and small tyrosine-kinase inhibitors (lapatinib).

\section{Conclusion}

Risk scores are extraordinarily valuable tools, highly recommendable in the clinical practice. However, their real use needs to be redefined and most importantly, we need to have their particular conditions of use in mind together with their limits and misinterpretation risks.

The main strengths of the present study are the big sample size of patients included in this national database (which constitutes an exceptional reflect of MBC patients diagnosed and treated in Spain), because we have considered factors at the time of recurrence and at the time of first diagnosis. The best endpoint to consider in long-term follow-up studies is survival. In our experience, we focused on OS and tried to set up the estimated probability at 12,36 and 60 months, thanks to the long-term follow-up available for these patients that let us analyze a real survival measurement, rather than a projected survival.

The study of prognostic factors in recurrent breast cancer is a clinically relevant field of development, from a biological but also from a clinical point of view. Large population databases with a long-term follow-up are an excellent source of clinical information that reflects the real clinical practice outside clinical trials. Our study will be helpful for better stratification and more accurate predictions of survival in the metastatic breast cancer (MBC). Focusing on overall survival we tried to estimate the probability of death at 12,36 and 60 months, constructing 
a prognostic index model to be further validated in another set of patients and possibly completed with new molecular markers in future Alamo projects.

\section{References}

1. Jemal A, Siegel R, Ward E et al (2008) Cancer statistics 2008. CA Cancer J Clin 58:71-96

2. López-Abente G, Pollán M, Aragonés N et al (2003) Plan Integral del Cáncer: Situación del Cáncer en España. Ministerio de Sanidad y Consumo, Madrid

3. Hortobagyi GN (1998) Treatment of breast cancer. N Engl J Med 339:974-984

4. Greenberg PA, Hortobagyi GN, Smith TL et al (1996) Long-term follow-up of patients with complete remission following combination chemotherapy for metastatic breast cancer. J Clin Oncol 14:2197-2205

5. Surveillance, Epidemiology, and End Results (SEER) Database. Available at: http://www.seer.cancer.gov/statfacts/htlm/breast. htlm

6. Hudis CA (2003) Current status and future directions in breast cancer therapy. Clin Breast Cancer 4:S70-S75

7. Carter CL, Allen C, Henson DE (1989) Relation of tumor size, lymph node status, and survival in 24,740 breast cancer cases. Cancer 63:181-187

8. De la Haba-Rodríguez J, Aranda E, Llombart A et al (2008) Time-to-progression in breast cancer: a stratification model for clinical trials. Breast 17(3):239-244
9. Insa A, Lluch A, Prosper F et al (1999) Prognostic factors predicting survival from first recurrence in patients with metastatic breast cancer: analysis of 439 patients. Breast Cancer Res Treat 56:67-78

10. Martín M, Mahillo E, Llombart-Cussac A et al (2006) The "El Álamo" project (1990-1997): two consecutives hospital-based studies of breast cancer outcomes in Spain. Clin Transl Oncol 8(7):508-518

11. McShane LM, Altman DG, Sauerbrei W et al (2006) Reporting recommendations for tumor marker prognostic studies (REMARK). Breast Cancer Res Treat 100:229-235

12. Vogel CL, Azevedo S, Hilsenbeck S et al (1992) Survival after first recurrence of breast cancer. The Miami experience. Cancer 70:129-135

13. Chang J, Clark G, Allred C et al (2003) Survival of patients with metastatic breast cancer. Importance of prognostic markers of the primary tumor. Cancer 97:545-553

14. Woodward W, Vinh-Hung V, Ueno N et al (2006) Prognostic value of nodal ratios in node-positive breast cancer. J Clin Oncol 24:1910-1916

15. James JJ, Evans AJ, Pinder SE et al (2003) Bone metastases from breast carcinoma: histopathological-radiological correlations and prognostic features. Br J Cancer 89:660-665

16. Atalay G, Biganzoli L, Renard F et al (2002) Clinical outcome of breast cancer patients with liver metastases in the anthracyclinetaxane era. Breast Cancer Res Treat 76:S47

17. Alba E, Ribelles N, Sevilla I et al (2001) Adjuvant anthracycline therapy as a prognostic factor in metastatic breast cancer. Breast Cancer Res Treat 66:33-39

18. Singletary SE, Walsh G, Vauthey JN et al (2003) A role for curative surgery in the treatment of selected patients with metastatic breast cancer. Oncolgist 8:241-251 